\title{
Progressive Non-rigid Registration of Temporal Mesh Sequences
}

\author{
Wieland Morgenstern \\ Fraunhofer HHI
}
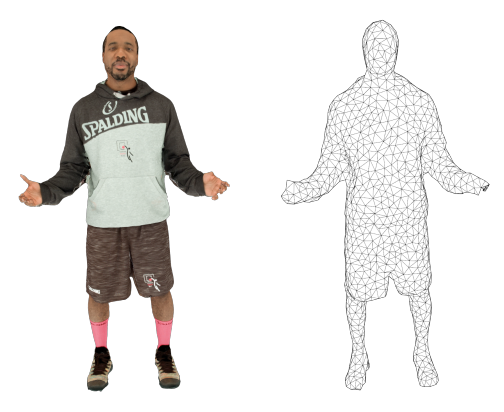

\author{
Anna Hilsmann \\ Fraunhofer HHI
}

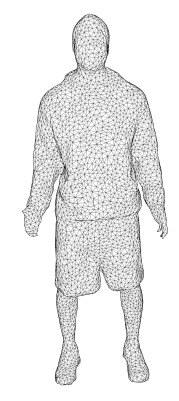

\author{
Peter Eisert
Fraunhofer HHI \\ Peter Eisert
Fraunhofer HHI
}

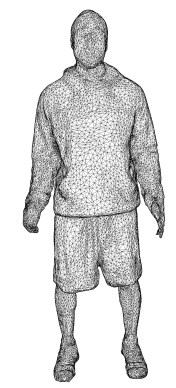

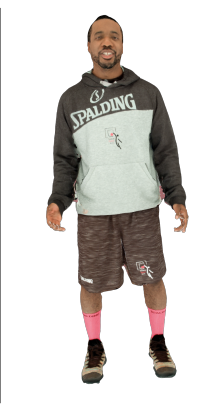

Figure 1: The mesh on the left is deformed in a coarse-to-fine approach to closely approximate the surface of the target mesh after the divider on the right.

\begin{abstract}
We present an algorithm for progressive mesh registration to provide temporal consistency, simplify temporal texture editing and optimize the data rate of 3D mesh sequences recorded in a volumetric capture studio.

The capture pipeline produces a sequence of manifold meshes with varying connectivity. We split the sequence into groups of frames that will share connectivity and chose a keyframe in each group that is progressively deformed to approximate the surface of the adjacent meshes. Our algorithm works with a coarse-to-fine ICP approach, which makes it robust against large deformations in the scene while preserving small details. Applying a deformation graph constrains the transformations to be locally as-rigid-as-possible, while allowing it to work with any natural objects in the scene, not just humans.

We show how to robustly track sequences of human actors with varying clothing over hundreds of frames recorded in a volumetric capture studio. We verify our results with a publicly available dataset of more than 40,000 frames. Our mesh registration takes less than five seconds per frame on a single desktop machine and has been successfully integrated into a volumetric capture pipeline for commercial use.
\end{abstract}

\section{CCS CONCEPTS}

- Computing methodologies $\rightarrow$ Volumetric models; Shape representations; Shape inference; Reconstruction; Mesh geometry models.

\section{KEYWORDS}

mesh registration, surface tracking, non-rigid alignment

Permission to make digital or hard copies of part or all of this work for personal or classroom use is granted without fee provided that copies are not made or distributed for profit or commercial advantage and that copies bear this notice and the full citation on the first page. Copyrights for third-party components of this work must be honored

For all other uses, contact the owner/author(s).

CVMP '19, December 17-18, 2019, London, United Kingdom

(c) 2019 Copyright held by the owner/author(s).

ACM ISBN 978-1-4503-7003-5/19/12.

https://doi.org/10.1145/3359998.3369411
ACM Reference Format:

Wieland Morgenstern, Anna Hilsmann, and Peter Eisert. 2019. Progressive Non-rigid Registration of Temporal Mesh Sequences. In European Conference on Visual Media Production (CVMP '19), December 17-18, 2019, London, United Kingdom. ACM, New York, NY, USA, 10 pages. https://doi.org/10.1145/ 3359998.3369411

\section{INTRODUCTION}

As virtual and augmented reality devices are becoming ubiquitous, there is a growing need to provide immersive 3D content. Applications like e-learning and entertainment benefit from working with natural human representations. Volumetric capture studios give us the option to capture actors in a natural environment, recording their minute facial expressions and life-like interactions with props.

A volumetric capture studio records in full 360 degrees with cameras aligned roughly on a sphere around the scene. The capture pipeline produces a sequence of 3D surface meshes. Frames are processed individually, thus while the scene changes gradually over time, the mesh connectivity may be different from frame to frame. While the mesh structure is invisible when rendered with a texture, the vertices of neighboring frames may sample the object surface differently. Editing textures over multiple consecutive frames editing is hindered by the texture atlas changing from frame to frame.

The system presented in [Ebner et al. 2017] captures natural scenes with 32 cameras at $30 \mathrm{fps}$. With each camera sensor providing a high-resolution $20 \mathrm{MP}$ image, the reconstructed scenes are highly detailed. The high level of detail is beneficial to the immersion, yet storage and transmission are expensive. Low-powered $\mathrm{VR} / \mathrm{AR}$ devices may struggle to ingest and display the data at its full resolution and frame rate.

In this work, we provide a way of reducing the amount of data and providing temporal consistency, by keeping the same mesh connectivity stable over a group of frames. A consistent topology over several frames allows for better compression of the volumetric video stream, as the connectivity does not have to be encoded for every frame. Changes to the geometry can be expressed as changes 
to the vertex coordinates, which have correspondences from frame to frame. Furthermore, using the same texture atlas over several frames allows for the texture images to be encoded as a video stream instead of single images. Editing consecutive frames in the texture space is facilitated by the texture atlas staying constant within the mesh connectivity group.

The task of registering one mesh to another is difficult, as natural scenes may contain a wide range of object shapes and temporal sequences allow for many possible deformations of these objects. Noisy measurements and imperfect reconstructions can further complicate the task.

We select a number of frames from the sequence to form keyframes, which are not processed further. We then deform the chosen keyframes to match the surface of their neighboring meshes, progressively forward and backward in the sequence. A subset of frames midway between keyframes is registered from both surrounding keyframes. By choosing the resulting mesh with the lower registration error, we ensure a smooth transition between keyframes.

Our pairwise mesh registration is based on the iterative closest point (ICP) algorithm. We present a hierarchical approach, to speed up the convergence toward a global solution even with large displacements between successive frames while preserving high-level detail. The deformation of a mesh is encoded as transformations on a deformation graph that grows in detail in this hierarchical approach. The deformation graph is kept as-rigid-as-possible to preserve local stiffness in articulated objects.

Given this approach, we can enforce local temporal consistency in long mesh sequences recorded in a volumetric capture studio. Our algorithm is robust against noisy recordings and is not limited to, but works well with, recordings of humans.

\section{BACKGROUND}

Non-rigid registration of 3D objects exists in many possible variants as the data used can be point clouds, partial views or full volumetric meshes. Due to the high degree of freedom given by correspondence choice and local transformations, the problem is ill-posed when formulated without further constraints. A good overview of registration approaches of $3 \mathrm{D}$ point clouds and meshes, both rigid and non-rigid was compiled by [Tam et al. 2013].

Human motion can be captured from the scene by fitting human models [Bogo et al. 2017][Fechteler et al. 2019] onto meshes of the human body. Recent contributions work with outdoor sequences [Robertini et al. 2016] and demonstrate real-time processing [Habermann et al. 2019].

Without modeling humans explicitly, a generic template can be trained from a rest pose [Kausch et al. 2017] or a sequence of meshes [Li et al. 2009][Zollhöfer et al. 2014]. This requires the subject in the scene to be topologically consistent throughout the sequence, which is often not given in natural scenes, due to human interaction with elements of the scene.

To support changing topology over time, the sequences can be split up into subsequences [Collet et al. 2015], with one mesh chosen as the keyframe per subsequence. Tracking can also be performed non-sequentially by identifying similar shapes throughout the sequence or even across sequences and registering the meshes according to the shortest path through a globally constructed similarity tree [Budd et al. 2013].

Sumner et al. developed a deformation graph to allow users direct manipulation of different shape representations (triangle meshes, polygon soups or particle simulations)[Sumner et al. 2007]. Each node in the graph has an affine transformation associated with it, and the nodes are connected by undirected edges, implying their overlapping influence on the target shape. The as-rigid-as-possible (ARAP) surface modeling by Sorkine and Alexa solves a similar problem, providing an iterative mesh editing scheme based on locally rigid transformations [Sorkine and Alexa 2007]. Locality is created from overlapping cells which are defined as the set of triangles incident upon each vertex, with similarities to the Laplacian mesh representation [Sorkine et al. 2004]. This cell structure is tied to the mesh resolution, whereas the deformation graph can have a resolution independent of the number of vertices in the deformed mesh. While they devise a way to compute optimal rotation, the ARAP framework provides an alternative minimization strategy, instead of global optimization over all parameters.

$\mathrm{Li}$ et al. presented a robust pairwise registration framework that simultaneously solves for point correspondences and surface deformation with a single global optimization [Li et al. 2008]. The $3 \mathrm{D}$ correspondences are parameterized in the $2 \mathrm{D}$ coordinates of the depth map. This approach automatically constrains the points to lie on the target scan and avoid the re-projection of the iterative closest point algorithm. While this works well for stereo camera registration, an application to volumetric meshes would require the definition of local parameterizations over the mesh, which is not given.

In follow-up work, Li and colleagues acquire a template of an object while keeping it rigid [Li et al. 2009]. The template mesh is low-pass filtered to reduce transient features. After the rigid template is acquired, dynamic acquisitions of partial scans are warped onto the template to create a reconstructed shape. As the template mesh is smoothed to reduce transient features, an additional step of detail synthesis is needed to recover dynamic detail. [Zollhöfer et al. 2014] demonstrated that this approach can be modified to run in real-time. Varanasi et al. show the ability to construct a dense displacement field from sparse photometric and geometric matches through Laplacian diffusion [Varanasi et al. 2008]. Wei et al. introduce a deep learning framework that provides dense correspondences between 3D scans of people [Wei et al. 2019].

Collet et al. describe an end-to-end volumetric capturing solution that includes mesh tracking. They segment the recorded performance into sequences of deformed keyframe meshes [Collet et al. 2015]. The keyframes are tracked forward and backward until an error threshold is surpassed, then another keyframe is greedily chosen, which complicates a scheme to parallelize the processing. Running on a single machine, the algorithm takes several minutes to register one mesh pair.

We propose a novel coarse-to-fine approach for pairwise mesh registration, where the deformation graph is directly extracted from the mesh, thus keeping references to the vertices it will transform. We do not perform a template acquisition over a range of meshes. Instead, we start the registration directly from the keyframe. 

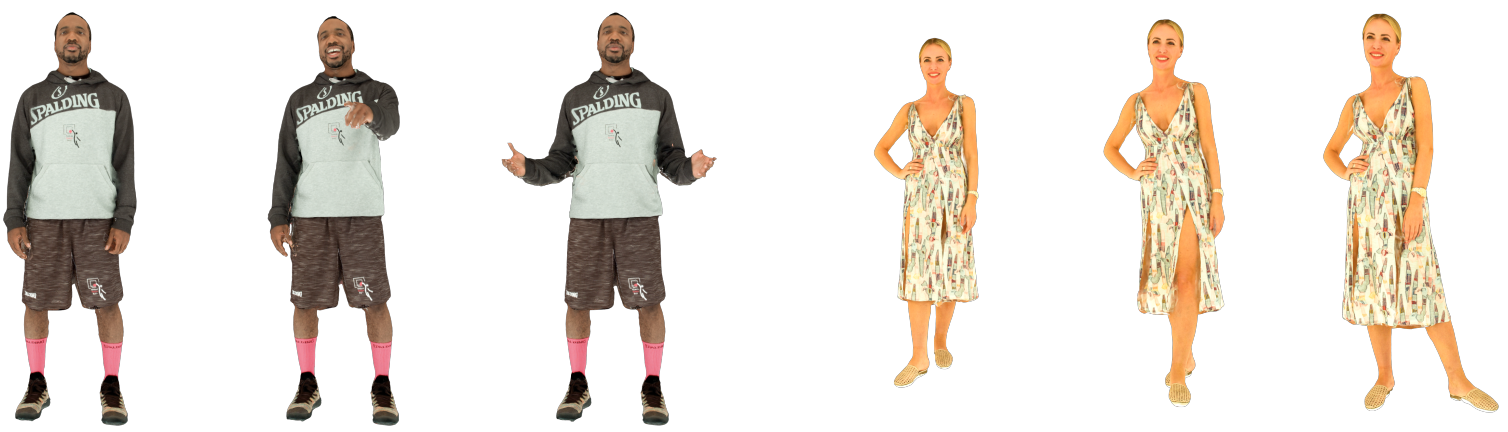

Figure 2: Examples from the Josh and the Lana mesh sequences used in our experiments.

We work in a block-based frame scheme to get a predictable keyframe rate. Instead of the user indirectly determining the number of keyframes through an error threshold as in [Collet et al. 2015], we chose a direct approach of allowing the user to set the average keyframe rate and specify additional keyframes if needed. Frames are tracked from two directions and meshes are registered from two different keyframes. Greedily selecting a winner reduces the local registration error and ensures a smooth transition between keyframes.

We parameterize rotations as quaternions, which reduces the number of total parameters in the optimization. By constraining the rotations to unit quaternions, we eliminate the need for a rotation error that enforces orthogonal columns in the rotation matrices used by [Sumner et al. 2007]. We relax the rigidity implicitly by adding more nodes to the deformation graph, which reduces the rigidity neighborhood size, rather than relaxing the regularization weights by halving them between iterations [ $\mathrm{Li}$ et al. 2009].

The progressive registration scheme and parallelization from block-based processing reduces the computation time to make this usable even for sequences captured with thousands of frames on a single high-end desktop machine.

\section{MESH SEQUENCE TRACKING}

The volumetric capture pipeline produces a sequence of triangular meshes $M_{t}$ with $t \in\left\{t_{1}, \ldots, t_{z}\right\}$. Each mesh consists of a set of vertices $V_{t}$, a set of edges $E_{t}$ and a set of faces $F_{t}$, forming $M_{t}=$ $\left(V_{t}, E_{t}, F_{t}\right)$. The mesh geometry is given through the set of vertex coordinates $V_{t}=\left\{v_{i} \in \mathbb{R}^{3} \mid i=\left\{1, \ldots, N_{t}\right\}\right\}$.

As frames are produced independently during the capture process, the connectivity $\left(E_{t}, F_{t}\right)$ of a mesh at time $t$ may be substantially different from the connectivity of the following frame, $\left(E_{t+1}, F_{t+1}\right)$. Through the mesh sequence tracking we aim to give neighboring meshes shared connectivity, differing only in their geometries $V$. Meshes in the captured sequence are transformed to form a tracked sequence that is visually indistinguishable when rendered with textures, but requires less data when stored due to the shared connectivity.

In the following sections, we describe how to choose groups of frames to share the same connectivity and then schedule the pairwise registration, starting from the selected keyframes.

\subsection{Keyframe Selection}

Large changes to the scene throughout a sequence can make it infeasible to keep the same connectivity for the complete sequence, which may run up to hundreds or thousands of frames. We adapt the keyframe concept from video compression and divide the sequence into groups of frames to share the same connectivity. The mesh sequence tracking begins from a keyframe within each group of frames. In later sections, we describe how we deform the mesh of the keyframe into its neighboring frames through pairwise registration.

The chosen keyframe must be able to represent all neighboring frames through deformation. If the neighboring frames can not be represented by the deformed keyframe, for example if new objects enter the scene, there will be a residual registration error, which we want to avoid. Selecting keyframes automatically requires a good selection criterion that predicts the mesh registration error. A keyframe feasibility score is presented in [Collet et al. 2015], where a high score per frame is achieved by the mesh $M_{t}$ having a high number of connected components $C_{t}$ and each connected component $c \in C_{t}$ having

- a high surface area $A_{c}$

- a low genus $g_{c}$

Using $g_{\max }$ and $A_{\max }$ as the maximum genus and surface of all meshes, the keyframe score $s_{t}$ is computed as

$$
s_{t}=\sum_{c \in C_{t}}\left(1+\left(g_{\max }-g_{c}\right)+\frac{A_{c}}{A_{\max }+1}\right)
$$

To achieve robust results from this scoring, the meshes need to contain low topological noise, such as tunnels, handles or holes. Otherwise the high genus will result in biased frame scores.

Additional constraints have to be considered when the sequences are meant to be rendered in real-time. Rendering on low-end devices like standalone virtual reality headsets pose requirements on keyframe frequency:

- an average distance, otherwise upload of data from RAM to GPU could exhaust available memory bandwidth, especially on low-end/mobile devices

- a minimum distance, otherwise bandwidth problems may arise from unexpected bursts 

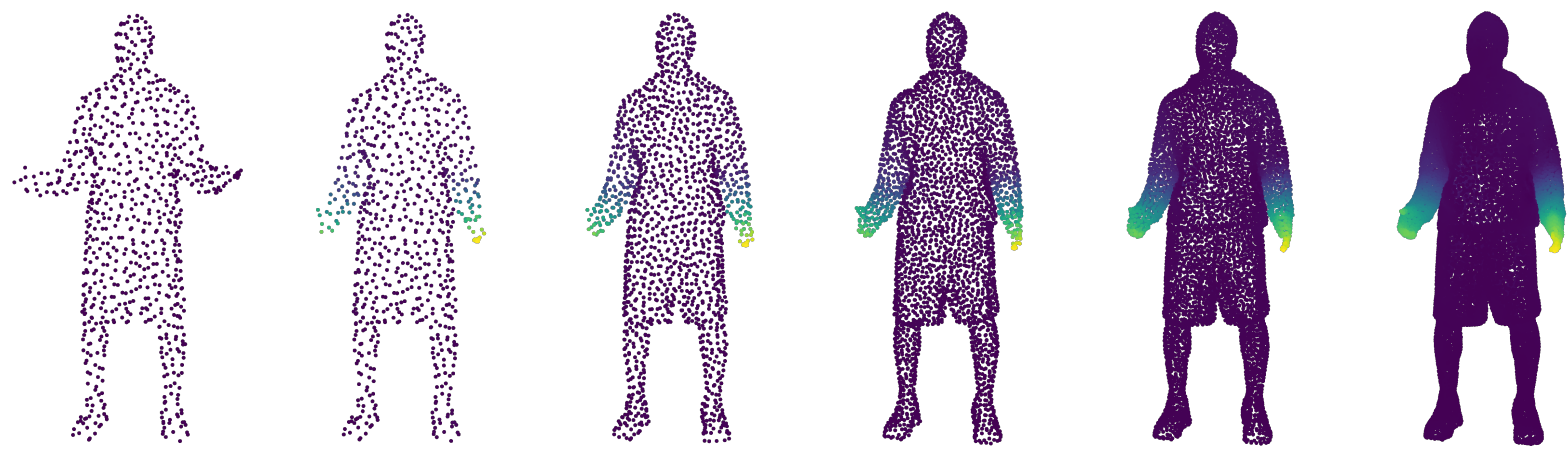

Figure 3: Translation magnitude of the nodes of the deformation graph, the magnitude is normalized per graph. Shown are stages from the registration of frames showing a person that moves their hands downward. The nodes close to moving body parts will have large translations associated with them, while the rest of the body stays still.

Increasing the distance between keyframes is beneficial to sequential real-time rendering and texture editing. Whether it improves the compression rate depends on the mesh compression algorithm, which may have a limited lookahead window and would insert additional keyframes. Large keyframe distances hinder random access of frames in compressed sequences. Under these considerations, we choose to split the mesh sequence into blocks (or subsequences) of length $n$, the keyframe step size. From each block, the frame with the highest keyframe feasibility score $s_{t}$ is chosen as the local keyframe. If the keyframe feasibility score is considered too unreliable for a scene as there is too much noise present, the user has the option to pick every nth frame as a keyframe instead.

In practice, we found keyframe step sizes of 15 to 25 frames to produce satisfactory results. We explore the registration error under different keyframe step sizes in the experiments in section 5.1. If there are severe topology changes between selected keyframes, like an object connecting with another object or the creation and destruction of a hole, the registration may not be satisfactory. We found it helpful to give the human operator, often the digital artist working on the scene, the option to manually specify additional keyframes when working with real-world data.

\subsection{Mesh Registration Scheduling}

After splitting the sequence into groups and choosing one keyframe per group, we now describe the order of pairwise registrations that are performed, given the list of keyframes. We start with any keyframe mesh $M_{t}$ at time $t$, which becomes the source for the following registrations. The first target mesh is $T=M_{t+1}$. The goal of the pairwise registration, which is described in detail in the following section, is to transform the vertex coordinates of $S=M_{t}$ (the source) so that its surface matches the surface of the target mesh $T$. The registered mesh $S^{\prime}=M_{t \rightarrow t+1}$ retains the connectivity of the source mesh $S$. In the next step, $M_{t \rightarrow t+1}$ will become the source mesh $S$ and is registered onto the target $T=M_{t+2}$.

With every registration, there is an error remaining, as the topology of the source frame may not perfectly fit the target frame. This error is likely to increase as the registration moves further away from the keyframe, due to movements in the reconstructed scene. In addition to the registration going temporally forward as described, the registration can also work backward, registering $M_{t}$ onto $M_{t-1}$. Every frame $M_{t}$ between two keyframes $M_{k}$ and $M_{j}$, with $k<t<j$ could be registered from either keyframe. By transitioning from one keyframe to the next midway between two keyframes, we cut the average temporal distance of each registered frame to its keyframe in half. As the registration process runs offline, we can work backward without any buffering while still producing a coherent output mesh sequence that can be rendered in order.

The frames are scheduled to be registered from the direction of their closest keyframe. For each group, one frame that is equidistant to two keyframes is chosen to be registered twice, from both forward and backward keyframes. Only the resulting mesh with the lower registration error is kept. Registration continues from the winning mesh along its original direction. The result is then compared to the previously computed frame from the other direction. The process continues until the registration error of the newly computed mesh is no longer lower than the previously computed one or the next keyframe is reached.

Additionally to smoothing the transition between keyframes, this approach supports large topology changes that can only be represented in either keyframe. Meshes created with a large error by registering from in one direction may all be replaced by meshes created from the keyframe of the other direction. This reduces the potential errors created from imperfect keyframe selection. While the process does not change the number of keyframes, it may lead to switching intervals between keyframes being irregular. But there can never be more than two consecutive connectivity changes or more than two connectivity changes in any group of frames during the length of the keyframe step size.

As the keyframes are chosen before any registration is performed, the registrations of different frame groups can run in parallel. Given $k$ keyframes, there can be $2 k$ concurrent mesh computations, as the registration starts both forward and backward from every keyframe. Computations need to be synchronized when transitioning between keyframes. The degree of concurrency can drop towards the end of the process, as the computation branches may take varying lengths of time to finish. The work is finished when all groups are processed and every mesh in the sequence was registered from at least once keyframe. 

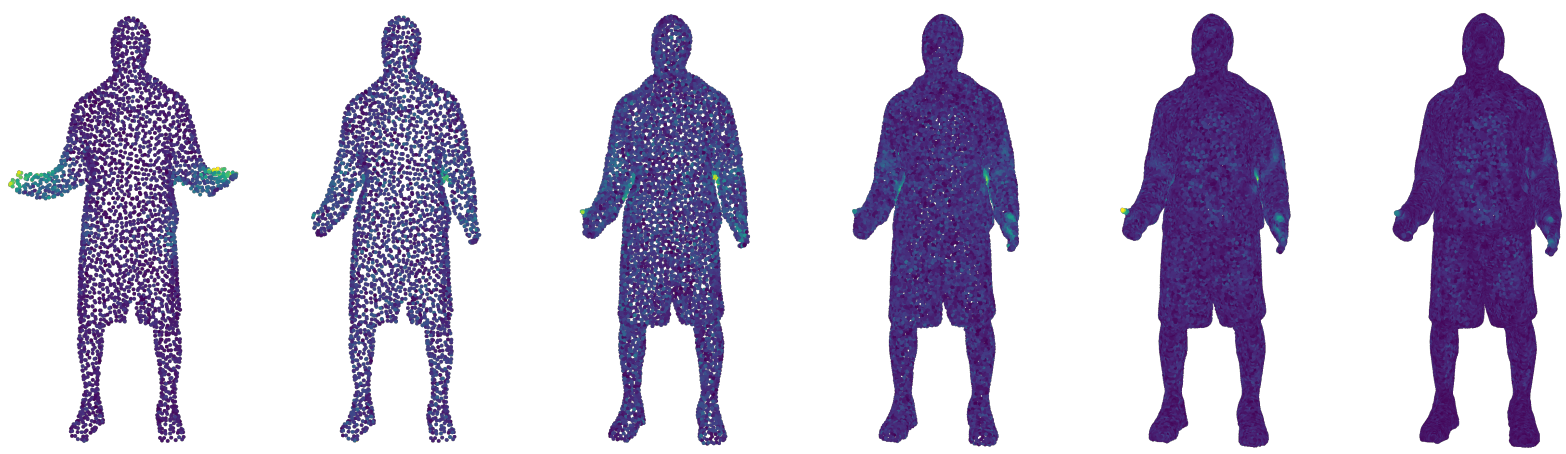

Figure 4: Magnitude normalized per mesh of the source-to-target residuals at various stages of the algorithm. Early on, the residuals are concentrated in the non-matching body parts and get spread out over the complete body as the optimization converges.

\section{PAIRWISE REGISTRATION}

The keyframe selection described in the previous section yields a schedule of pairwise registrations. At each step, a mesh $S$ is deformed to closely approximate the surface given by its neighboring $T$, into $S^{\prime}$. The first $S$ to be deformed will be a keyframe, while the following registrations will start from the already processed neighbor, propagating the deformations throughout the sequence. Textures for all but the keyframes are discarded in the registration process, as their texture atlases no longer apply to the registered mesh. Textures for the registered meshes must be recreated after the registration process.

We apply a coarse-to-fine scheme in the deformation. Initially, a low-resolution mesh is deformed, then progressively more details are added and the deformation is refined. Figure 1 shows meshes at various stages throughout the registration. At each resolution, we run a bidirectional iterative closest point algorithm to pull the mesh towards the target surface while constraining it to be locally as-rigid-as-possible.

\subsection{Multi-Resolution Scheme}

Our non-rigid registration approach is based on the iterative closest point (ICP) algorithm [Besl and McKay 1992]. The deformation process is computationally expensive when using all samples from a high-resolution mesh, due to the correspondence matching and non-linear optimization. The number of ICP iterations using the complete set of samples can be reduced by applying a coarse-to-fine strategy, as demonstrated in [Jost and Hugli 2002]. The ICP can first run on a small subset of samples, which is computationally relatively cheap and then improve over several levels of resolution. While the ICP still needs to run on the full-resolution mesh to register high-frequency detail, it has to run fewer iterations, as the global deformation is already found.

As our registration enforces local rigidity, we require a connected mesh at every resolution, not just a set of $3 \mathrm{D}$ points. A multi-resolution mesh pyramid needs to be constructed. The progressive mesh representation introduced in [Hoppe 1996] shows how to create meshes at different resolution levels by progressively collapsing individual edges.
We chose to build a resolution pyramid from the mesh at different levels of detail. The highest level of the pyramid is initialized with the original mesh. The next lower level is created by reducing the mesh to contain half the number of faces. The lowest level mesh is enforced to have at least 1024 vertices, the mesh is then not further reduced.

A mesh $M^{\text {high }}$ is reduced into half the resolution $M^{\text {low }}$ through an iterative edge collapse approach. $M^{l o w}$ is initialized as a copy of $M^{h i g h}$. At every step, the shortest remaining edge from vertex $v_{i}$ to $v_{j}$ is collapsed into its midpoint $v_{\text {mid }}=\left(v_{i}+v_{j}\right) / 2$, until the number of faces in $M^{l o w}$ is equal or less than half the number of faces in $M^{\text {high }}$. Similar to the progressive mesh representation [Hoppe 1996], we record the indices of the involved vertices in every edge collapse. The collected records allow us to retrieve a reference of each vertex $v_{i}$ in $M^{\text {high }}$ to a vertex $v_{j}^{\prime}$ in $M^{l o w}$. These references are key to transferring parameters determined for $M^{l o w}$ onto $M^{\text {high }}$ in the coarse-to-fine process.

We create a progressive mesh pyramid for both source mesh $S$ and target mesh $T$ to perform all registrations between meshes of similar resolution. We begin the registration by estimating the deformation of the coarsest level of $S$ onto the coarsest level of $T$. When the closest point algorithm converges, the deformation parameters are transferred to the source mesh of the next-highest level using the recorded vertex index references. The weighted averaging scheme described in the following section is applied when computing the parameters of the graph on the upper level. Having upsampled the parameters to the next level, the registration is started again. This continues until the full resolution mesh is registered onto its full-resolution target.

Our deformation algorithm requires the deformable mesh to be manifold. Non-manifold components can appear in the input data from the Poisson meshing. By reviewing our recorded data, we found that these components appear exclusively on object edges and don't contain reliable texture data, thus we chose to delete all non-manifold components from the mesh without trying to repair the mesh around the broken components. Faces that contain nonmanifold edges and faces that contain non-manifold vertices are removed from all meshes involved in the registration. 


\subsection{Deformation Graph}

We want to be able to capture arbitrary objects in the volumetric capture studio. Most scenes do contain a human actor, but they may be dressed in a way that can make it difficult to infer the body shape. They often interact with items, picking up or dropping objects, which makes it impossible to create templates of the scene that are valid for the complete sequence. Meshes may merge or split over time. Hence, we need a more general approach to deformation.

We model the deformation of the object with a deformation graph akin to the version presented in [Sumner et al. 2007]. The deformation graph is an extended mesh with a deformation node at every vertex position of the mesh. Each node defines an affine transformation as a representation of the deformation of mesh vertices in its proximity: a unit rotation quaternion $q_{n}$ and a $3 \times 1$ translation vector $t_{n}$.

The deformation graph node describes a transformation that will be applied to nearby points. A point $p$ in the proximity of node $p_{n}$ is translated and rotated around $p_{n}$ into $p^{\prime}$ :

$$
p^{\prime}=p_{n}+q_{n} \cdot\left(p-p_{n}\right) \cdot q_{n}^{*}+t_{n}
$$

We define $q_{n}^{*}$ as the inverse rotation of $q_{n}$. When rotating a point $r \in \mathbb{R}^{3}$ in shorthand $r^{\prime}=q_{n} \cdot r \cdot q_{n}^{*}$, we imply the construction of a quaternion from the coordinates of $r$, and the extraction of the coordinates into $r^{\prime}$ from the resulting quaternion: $\left(0, r_{x}^{\prime}, r_{y}^{\prime}, r_{z}^{\prime}\right)=$ $q_{n} \cdot\left(0, r_{x}, r_{y}, r_{z}\right) \cdot q_{n}^{*}$

As we can see, transforming the node itself would simply apply the translation term: $p_{n}^{\prime}=p_{n}+t_{n}$.

We want to achieve a smooth deformation of the mesh, so the transformation of each point on the mesh is a weighted average of the transformation of neighboring deformation graph nodes. There is no direct relationship between the points on the mesh and the nodes of the graph. Selecting the nodes with the smallest Euclidean distance might result in inaccurate correspondences since vertices of surfaces pointing in opposing directions could be selected. We would prefer a geodesic distance that matches connected components on the shortest path.

As described in the previous section, the nodes of the deformation graph were constructed through decimation of the mesh, while keeping references from graph nodes to the mesh vertex at the original resolution. Through a reverse lookup of these references, we find the node $n$ that $v_{i}$ was collapsed into. We take the nodes adjacent in the deformation graph, connected over at most two edges. From the node $n$ and its neighbors, we pick the $k=3$ with the smallest Euclidean distance to $v_{i}$.

Of all $k=3$ neighboring nodes we compute the maximum distance $d_{\max }$ to $v_{i}$. Each weight $w$ is computed as

$$
w=\left(1-\frac{v_{i}-p_{n}}{d_{\text {max }} * d_{\text {scale }}}\right)^{2}
$$

The value of $d_{\text {scale }}$ can be chosen to any factor larger than 1 . Using this fixed max scaling allows us to forgo finding an additional $k+1$ neighbor for normalization. We have used $d_{\text {scale }}=1.2 \mathrm{in}$ our experiments and did not notice a degradation in performance compared to normalizing with the distance to an additional fourth neighbor.

The transformation of a point $p$, using the smooth deformation from the neighboring parameters is written as

$$
p^{\prime}=\sum_{n=0}^{3} w_{n}\left(p_{n}+q_{n} \cdot\left(p-p_{n}\right) \cdot q_{n}^{*}+t_{n}\right)
$$

The mesh from the next lowest level becomes the deformation graph of the current level. The lowest mesh level uses a deformation graph again decimating to half its size given the above instructions. The magnitude of the transformation associated with the deformation graph nodes is plotted in Figure 3. The deformation graph is initialized without any translation and quickly picks up the global deformations on the low-resolution levels, refining the local transformations on the higher detailed levels.

For every keyframe, a deformation graph pyramid is created. The parameters at the deformation graph nodes change throughout the optimization process, but the topology of the deformation graph is kept constant, creating temporal consistency around the keyframe.

\subsection{Optimization}
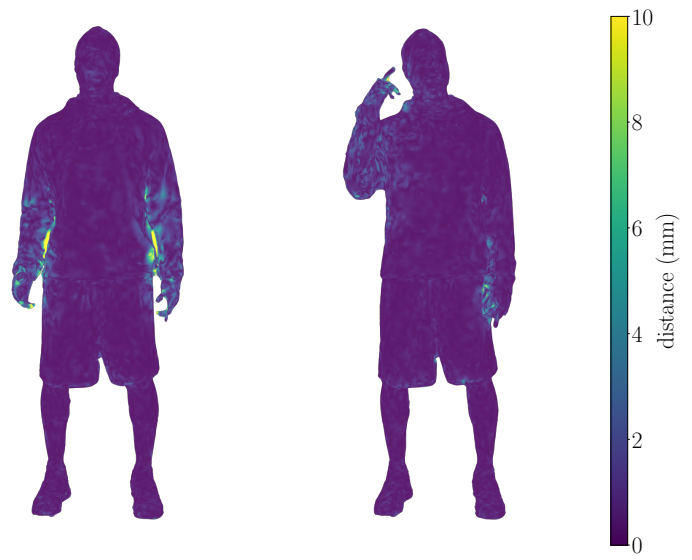

Figure 5: Registered meshes with the vertex color encoding the distance to the target mesh. We picked examples from the sequence where the arms start separating from the body. The amount of arm-to-body connection may differ from frame to frame, and some error residual remains trying to register the meshes of different connectivity.

Given a source mesh $S$, a target mesh $T$ and a deformation graph $D$, the goal is to find the transformation parameters $q_{n}, t_{n}$ for all nodes of $D$ that allow for the best approximation $S^{\prime}$ of $S$ to $T$. Correspondences between $S$ and $T$ are found, and an optimization procedure will determine all $q_{n}, t_{n}$ to minimize the distance between all correspondences, while simultaneously keeping an as-rigid-as-possible structure.

As the structure to be deformed is a surface mesh and not just a point cloud, we have free choice over which points to sample from the surface to use in the ICP algorithm. We use the centroids of the mesh faces, which allows for a robust correspondence between source and target points if the meshes are of similar topology and are well-aligned. A vertex-to-vertex coordinate match may 
bounce between any of the adjacent triangles, whereas the centroidto-centroid match is more likely to pick the same triangle when introducing small adjustments to the coordinates.

For every face on the query mesh, we find a match of its centroid coordinate $c$ in the target mesh. A kd-tree is created from the target centroid coordinates. Using the nanoflann-library [Blanco and Rai 2014], the 16 nearest neighbors of each query centroid are retrieved from the tree. We chose the closest match that has its face normal pointing in the same hemisphere as the querying face. If none of the normals match, the closest match is chosen. This normalbased heuristic removes many possible mismatches of close surfaces pointing in opposite directions. In registrations of humans and other articulated objects, the heuristic helps to avoid mismatches between body parts held closely together, such as an arm next to the chest or individual fingers [Fechteler et al. 2019].

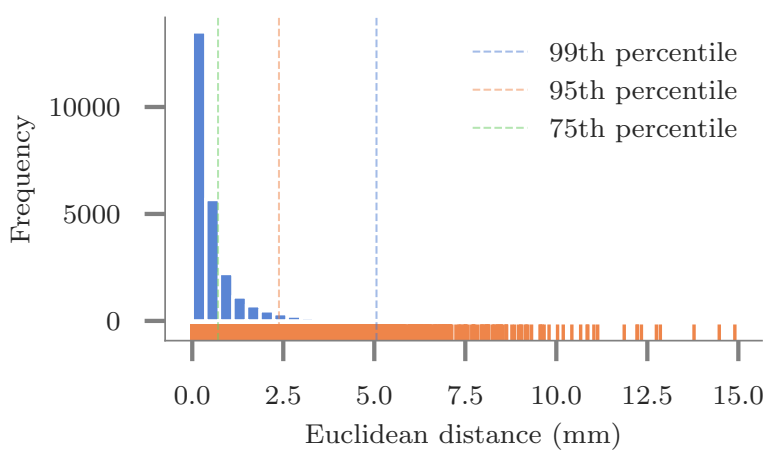

Figure 6: Histogram of the Euclidean distances between vertices of the registered mesh and the triangle surface of the target mesh for frame at $t=50$ in the Josh sequence. The rug plot (orange) below the histogram shows the scarcity of outliers beyond the 99th percentile.

If solely the correspondences from $S \rightarrow T$ were used in the optimization, a valid solution could be to contract parts of the mesh, not covering the complete surface of $T$. To counteract this behavior, we additionally compute correspondences from $T \rightarrow S$ which would increase the error if $T$ would not be completely covered by the registered mesh. Both sets of correspondences are used in the optimization problem.

As the objective function for the optimization we sum the different terms:

$$
E=\alpha_{S T} E_{S T}+\alpha_{T S} E_{T S}+\alpha_{\text {rigid }} E_{\text {rigid }}
$$

$E_{S T}$ and $E_{T S}$ are the data terms for the correspondences from source to target mesh and from target to source mesh.

Both can be inferred from a single data term

$E_{\text {dat } a}=\sum_{i=0}^{f}\left\|x_{i}-c_{i}^{\prime}\right\|=\sum_{i=0}^{f}\left\|x_{i}-\sum_{j=0}^{3} w_{i j}\left(c_{i}+q_{i j} \cdot\left(c_{i}-p_{i j}\right) \cdot q_{i j}^{*}+t_{i j}\right)\right\|$

$E_{S T}$ is constructed using $f$ centroids in the source mesh $S$, their coordinates $c_{i} \in \mathbb{R}^{3}$, and a correspondence $x_{i} \in \mathbb{R}^{3}$ on the target mesh $T$. For each centroid $c_{i}$ there are the three closest deformation graph nodes, with their fixed weights $w_{i j}$ computed through Equation (3) and the variable rotations $q_{i j}$ and translations $t_{i j}$.

The source-to-target residuals are displayed in Figure 4 over the course of the optimization of an example mesh. The largest residuals are reduced early on in low-resolution levels. Each additional resolution level reduces the error further by adding and aligning more details.

$E_{T S}$ is constructed using $f$ centroids from the target mesh $T$, their coordinates $c_{i}$, each centroids correspondence $x_{i}$ in the source mesh $\mathrm{S}$. The closest deformation graph nodes are found around $x_{i}$, which is in the source mesh, and their weights computed accordingly.

Given a deformation graph with $m$ nodes, $N(i)$ as the set of of nodes directly connected to node $i$ and the number of these neighbors $d_{i}=|N(i)|$, we write the rigidity regularization term as the difference between the node transforming itself and the average of the node being transformed with the parameters of its one-ring neighbors:

$$
E_{\text {rigid }}=\frac{1}{m} \sum_{n=1}^{m}\left\|p_{n}+t_{n}-\frac{1}{d_{n}} \sum_{i \in N(n)}\left(q_{n} \cdot\left(p_{n}-p_{i}\right) \cdot q_{n}^{*}+p_{i}+t_{i}\right)\right\|
$$

To find the optimal deformation, we minimize Equation 5 by modifying the quaternion rotation and translation on the graph nodes. We solve our non-linear least-squares problem with the LevenbergMarquardt algorithm implementation of the ceres library [Agarwal et al. [n. d.]]. We found the iterative Schur algorithm to work well as the linear solver for our sparse problem. We use the quaternion parameterization available in ceres to constrain all quaternions to unit rotation quaternions throughout the optimization process. The solver is terminated when $\Delta E \leq 10^{-5} E$.

In experiments we determined $\alpha_{\text {rigid }}=100$ and $\alpha_{S T}=\alpha_{T S}=1$ to enforce an appropriate stiffness for the scenes we recorded. We keep these weights constant for the complete registration process.

As the rigidity loss is enforced in the neighborhood of each deformation graph node, the rigidity loss becomes more local as more nodes are added to the graph progressively, resulting in a coarseto-fine optimization. The rigidity loss is scaled by the number of deformation graph nodes, whereas the data terms are not scaled by the number of points. Thus with every resolution increase, the total impact of the rigidity loss is halved, allowing for less restrained movement of the newly added points.

While we use the point-to-point distance of face centroid coordinates in the optimization, we check the average point-to-plane distance [Chen and Medioni 1992] matching the vertex coordinates on one mesh to the surface of the other mesh for the stop criterion. Using different samples and distance measures prevents overfitting in the ICP. We combine the mean distance over all vertices of the source-target and target-source directions into one number: $\operatorname{avgDist}=$ mean $_{S T}+$ mean $_{T S}$.

The ICP usually converges after a few iterations, it is forced to stop after at most 20 iterations. As a convergence criterion, we combine the change of average point-to-plane distance avgDist and the change of the total residuals, which include centroid-to-centroid distance and rigidity: 
Table 1: Quantitative description of the Josh and Lana mesh sequences used in the experiments

\begin{tabular}{ccc}
\hline & fosh & Lana \\
\hline Frames & 359 & 204 \\
Vertices & 37,600 & 46,500 \\
Edges & 150,000 & 195,000 \\
Faces & 50,000 & 65,000 \\
\hline
\end{tabular}

$$
\frac{E_{i-1}-E_{i}}{E_{i-1}}<0.01 \text { and } \frac{\operatorname{avgDist}_{i-1}-\text { avgDist }_{i}}{\text { avgDist }_{i-1}}<0.01
$$

The registration is complete when the ICP on mesh of the highest detail converges.

\section{EXPERIMENTS AND RESULTS}

We have implemented the algorithm as a post-processing step to run after a volumetric capture pipeline [Schreer et al. 2019], taking the mesh files as input and outputting a mesh sequence. To demonstrate the performance of our proposed technique, we conduct a series of experiments on two sequences, fosh and Lana. Table 1 lists the number of meshes per sequence and the average mesh resolutions. Example meshes from the sequences are shown in Figure 2.

\subsection{Evaluation of Mesh Distance}

To evaluate the performance of the registration regarding the approximation to the target mesh, we measure the Euclidean distance of the vertices of the registered mesh to the closest point of the closest triangle of the target mesh using the AABB implementation in libigl [Jacobson et al. 2018].

As shown in Figure 6, most vertices are closely aligned with the target surface, but not all vertices can be aligned perfectly. Due to noise in the capturing process, the reconstruction may contain highfrequency features that are not matched in the registered mesh. For example, the noisy temporal reconstruction of individual fingers will lead to mismatches in the registration process. Furthermore, some topology changes, especially objects separating, objects fusing and hole creation and filling, can not be correctly captured with a fixed-connectivity keyframe. These effects produce a long tail of vertices that are a few millimeters off their target surface.

Figure 5 plots the computed distance from the target mesh onto two different registered meshes. It is visible how the misregistered vertices with several millimeters of distance are constrained to areas with self-occlusion and topology changes.

To describe the long-tailed distribution of the distance measurements, we compute the median values and the median absolute deviation (MAD). As a quantitative description of the outliers, we provide the 99th percentile of the distances.

Figure 7 shows the progression of the percentiles of the Euclidean distance function over the fosh sequence with a keyframe distance of 20.3/4 of the vertices are continuously below $1 \mathrm{~mm}$ to their target surface, while the 99th percentile varies with the scene and crosses to $1 \mathrm{~cm}$. As the keyframes are copied to the output untouched, the distance at each keyframe (at $t=0,20,40, \ldots)$ is zero.

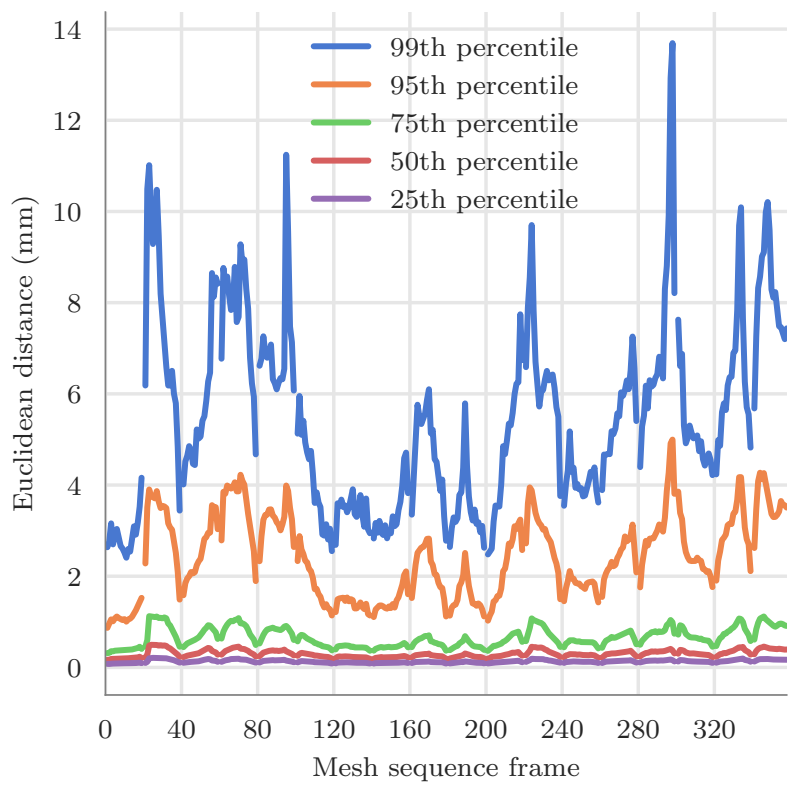

Figure 7: Statistical measures of the Euclidean distance between the vertices of the registered mesh and the triangle surface of the target mesh. The majority of vertices is in the sub-mm range at all times, while few outliers can go to several $\mathrm{mm}$ distance over the course of the sequence. The distance is zero for every 20th frame, which are the keyframes.

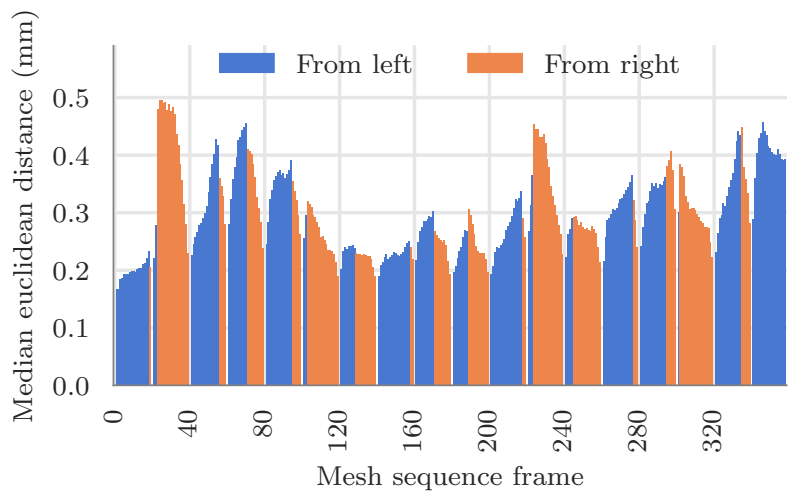

Figure 8: Median mesh distance vertex-to-surface of the registered frames to their targets over the Josh sequence given a constant keyframe step size of 20 frames. Frames registered from the keyframe to the left (earlier) marked blue, from right (later) marked orange.

Using a keyframe step size of 20, we can plot the median Euclidean distance of each registered mesh over a complete sequence. As shown in Figure 8, the median is constantly below $0.5 \mathrm{~mm}$ for the complete fosh sequence. As the registration is performed both forward and backward, the keyframe of any registered frame may be 
Table 2: Median distance, median absolute deviation (MAD) and 99th percentile Euclidean distance of all vertices of the Josh and Lana sequences, given different keyframe step sizes.

\begin{tabular}{l|ccc|ccc}
\hline $\begin{array}{c}\text { Step } \\
\text { size }\end{array}$ & median & MAD & $99 \%$ & median & MAD & $99 \%$ \\
\hline 5 & 0.240 & 0.486 & 4.879 & 0.166 & 0.340 & 3.444 \\
10 & 0.263 & 0.550 & 5.337 & 0.177 & 0.389 & 3.888 \\
20 & 0.289 & 0.615 & 5.719 & 0.187 & 0.416 & 4.047 \\
40 & 0.306 & 0.635 & 5.736 & 0.193 & 0.477 & 4.459 \\
80 & 0.330 & 0.719 & 6.355 & 0.196 & 0.461 & 4.324 \\
160 & 0.344 & 0.772 & 6.902 & 0.199 & 0.499 & 4.844 \\
\hline
\end{tabular}

to its left or its right. The graph shows that the keyframe transition rarely happens in the middle between two keyframes.

The keyframe step size can be varied. Table 2 lists the median distances, deviation and 99th percentile of all vertices in the sequence, given different keyframe frequencies. As all meshes in a sequence have roughly the same resolution, the distance statistics can be computed from all vertices in the sequence, regardless of which registered mesh they belong to. The keyframes are not considered in these statistics, as their distance to themselves is again all zero. The median registration error rises steadily with longer keyframe step sizes. As both sequences contain a single moving actor and no global changes, the registration error is tolerable even when using very few keyframes. This demonstrates the local rigidity scheme working well in deforming the mesh into different poses.

\subsection{Computation Time}

The registration of the fosh sequence of 359 meshes with a keyframe step size of 20 finished in 802 seconds, at a rate of 2.2 seconds per mesh. All experiments are run on a machine with 2 Intel Xeon Gold 6126 CPUs, computing up to 48 registrations in parallel. If the parallel mesh computation is disabled, the average registration time per mesh increases to 22 seconds. Due to the bidirectional registration and comparison, 473 meshes were created in total. Of the 114 extraneous meshes created for the same point $t$ from to different keyframes, 87 frames replaced the already computed mesh. The other 27 were immediately dropped, as they had a higher error than the previously computed mesh.

Additionally to performing the registration of meshes from different keyframes in parallel, we can use finer-grained parallelization in the pairwise mesh registration, in the correspondence finding, weight computation and parameter upsampling. Due to the nature of the coarse-to-fine process, the resolution levels have to be processed sequentially.

The mesh tracking approach presented by Collet and colleagues takes around 186 seconds (given there as $11 \%$ of the processing time of their capture pipeline, which they list as 28.2 minutes) on a machine with identical CPU core count [Collet et al. 2015]. Even though our models use a much higher resolution at $50 \mathrm{k}$ and $65 \mathrm{k}$ faces versus their $10 \mathrm{k}$ and $20 \mathrm{k}$ models, our computation time at 2.2 seconds per frame is nearly two orders of magnitude lower $(84 \mathrm{x}$ relative speedup).
Table 3: Median distance, median absolute deviation (MAD) and 99th percentile Euclidean distance of all vertices of the Dynamic FAUST subjects

\begin{tabular}{l|ccc}
\hline Subject & median & MAD & $99 \%$ \\
\hline 50002 & 0.230 & 0.429 & 3.914 \\
50004 & 0.190 & 0.454 & 3.820 \\
50007 & 0.226 & 0.419 & 3.949 \\
50009 & 0.247 & 0.415 & 3.608 \\
50020 & 0.176 & 0.318 & 2.820 \\
50021 & 0.188 & 0.362 & 3.154 \\
50022 & 0.207 & 0.447 & 4.174 \\
50025 & 0.200 & 0.358 & 2.952 \\
50026 & 0.233 & 0.428 & 4.006 \\
50027 & 0.242 & 0.461 & 3.744 \\
\hline
\end{tabular}

\subsection{Coarse-to-Fine}

The multi-resolution approach presented in section 4.1 was introduced to make the registration robust against large movements in the scene and to reduce the overall computation time, compared to an algorithm that would run all ICP iterations on the full-resolution mesh. Running a registration with a disabled coarse-to-fine approach of the fosh sequence with a keyframe step size of 20, we find that the median distance increases from $0.289 \mathrm{~mm}$ to $0.304 \mathrm{~mm}$. The additional registration error is comparable to using about half the number of keyframes, as the median error using the coarse-to-fine approach and a step size of 40 is comparable $(0.306 \mathrm{~mm}$, see Table 2 ). The computation time more than doubles ( $802 \mathrm{~s}$ to $1718 \mathrm{~s}$ ) when disabling the coarse-to-fine approach. Due to the missing rough alignment from lower levels, more iterations on the full-resolution level have to be run to converge, which are computationally much more expensive. This experiment demonstrates the effectiveness of the coarse-to-fine approach, both in registration quality and computation time.

\subsection{Automatic Keyframe Selection}

Applying the automatic keyframe detection scheme described in section 3.1, the median distance in the fosh sequence can be improved from $0.289 \mathrm{~mm}$ with a keyframe exactly every 20 frames to $0.283 \mathrm{~mm}$ with a keyframe on average every 20 frames, chosen through the keyframe feasibility score. If there is a risk of high amounts of noise in the sequence, the fixed keyframe step size may be preferred, as the keyframe feasibility algorithm would choose meshes with the highest topological noise, which could reduce the overall temporal consistency of the mesh sequence registration.

As the keyframe feasibility score computation does not require any registration, but simply analyses the geometry, it can be calculated quickly. For the fosh sequence, it takes around $15 \mathrm{~s}$ to compute the score for all 359 frames given the setup described in section 5.2.

\subsection{Performance on Dynamic FAUST Dataset}

We evaluate our algorithm on the MPI Dynamic FAUST dataset, which is publicly available [Bogo et al. 2017]. It contains different human subjects in motion at $60 \mathrm{fps}$. We reduce the resolution of the scans to a common 65,535 faces through an iterative edge collapse 
decimation. We keep only the largest connected mesh component in every mesh: while the meshes contain only one subject mesh, the ground or a background wall is sometimes visible in single frames. The dataset contains 129 dynamic performances of 10 different subjects, with 41,220 meshes in total.

As in the other experiments, we run the registration with a keyframe every 20 frames. The computation of all sequences runs in 65.5 hours, at a rate of 5.45 seconds per pairwise registration. The detailed results are available in Table 3 . Although we perform the registration without any knowledge of human bodies and their motion, we achieve a median error of only $0.2 \mathrm{~mm}$ while reducing the data rate of the mesh connectivity to $1 / 20$. We show that our registration works robustly, even when the data contains a good amount of noise, as in this dataset.

\subsection{Limitations}

High-frequency details in the target frame but not represented in the keyframe may not be recovered. Finer detail such as wrinkles and folds could be fused onto the registered mesh in a post-processing step, reducing the number of outliers in the registration error. This is a trade-off, as it reduces temporal consistency and makes mesh compression less efficient.

The keyframe step size needs to be adapted to match the speed of large topological changes in the scene. If keyframes are too far apart, some meshes may not be properly registered. If keyframes are too close, the benefits from the mesh tracking are reduced.

\section{CONCLUSION}

We have presented a system that progressively registers frames within a mesh sequence to provide temporal consistency. Our coarse-to-fine approach with as-rigid-as-possible deformations runs quickly while being robust against large movements in the scene. Our experiments demonstrate that we can register neighboring frames of human actors with sub-millimeter median precision. Humans and their movement are not explicitly modeled, allowing for the presented approach to work on any natural scene. Using a keyframe every $n$ frames, we achieve a reduction of the amount of data in the mesh connectivity and texture atlas to $1 / n$. The locally constant connectivity enables a temporal vertex coordinate compression and facilitates temporal texture editing.

While the user can set the keyframe distance or manually add additional keyframes, the tracking algorithm runs automatically on the given mesh sequence, requiring no manual intervention or threshold tweaking. It can be easily integrated into a volumetric capture pipeline, allowing for the results to be used in a much wider range of $\mathrm{AR} / \mathrm{VR}$ devices than the unprocessed sequences. Our algorithm runs around $80 \mathrm{x}$ faster than the implementation by Collet et al. while achieving a median registration error below one $\mathrm{mm}$ for the tested scenes.

\section{ACKNOWLEDGMENTS}

The fosh sequence was recorded with Volucap for use in the $M a$ gentaVR app. This research has received funding from the EU Horizon 2020 research and innovation program under grant agreement 762021 (CONTENT4ALL).

\section{REFERENCES}

Sameer Agarwal, Keir Mierle, and Others. [n. d.]. Ceres Solver. http://ceres-solver.org. Paul J Besl and Neil D McKay. 1992. A method for registration of 3-D shapes. IEEE Transactions on Pattern Analysis and Machine Intelligence 14, 2 (1992), 586-606.

Jose Luis Blanco and Pranjal Kumar Rai. 2014. nanoflann: a C++ headeronly fork of FLANN, a library for Nearest Neighbor (NN) with KD-trees. https://github.com/jlblancoc/nanoflann.

Federica Bogo, Javier Romero, Gerard Pons-Moll, and Michael J Black. 2017. Dynamic FAUST: Registering human bodies in motion. In Proceedings of the IEEE Conference on Computer Vision and Pattern Recognition, Vol. 6. 6233-6242.

Chris Budd, Peng Huang, Martin Klaudiny, and Adrian Hilton. 2013. Global non-rigid alignment of surface sequences. International fournal of Computer Vision 102, 1-3 (2013), 256-270.

Yang Chen and Gérard Medioni. 1992. Object modelling by registration of multiple range images. Image and vision computing 10, 3 (1992), 145-155.

Alvaro Collet, Ming Chuang, Pat Sweeney, Don Gillett, Dennis Evseev, David Calabrese, Hugues Hoppe, Adam Kirk, and Steve Sullivan. 2015. High-quality streamable free-viewpoint video. ACM Transactions on Graphics (TOG) 34, 4 (2015), 69.

Thomas Ebner, Ingo Feldmann, Sylvain Renault, Oliver Schreer, and Peter Eisert. 2017. Multi-view reconstruction of dynamic real-world objects and their integration in augmented and virtual reality applications. Fournal of the Society for Information Display 25, 3 (2017), 151-157.

Philipp Fechteler, Anna Hilsmann, and Peter Eisert. 2019. Markerless Multiview Motion Capture with 3D Shape Model Adaptation. In Computer Graphics Forum. Wiley Online Library, Wiley Online Library.

Marc Habermann, Weipeng Xu, Michael Zollhoefer, Gerard Pons-Moll, and Christian Theobalt. 2019. Livecap: Real-time human performance capture from monocular video. ACM Transactions on Graphics (TOG) 38, 2 (2019), 14.

Hugues Hoppe. 1996. Progressive meshes. In Proceedings of the 23rd annual conference on Computer graphics and interactive techniques. ACM, 99-108.

Alec Jacobson, Daniele Panozzo, and Others. 2018. libigl: A simple C ++ geometry processing library. https://libigl.github.io/.

Timothée Jost and Heinz Hugli. 2002. A multi-resolution scheme ICP algorithm for fast shape registration. In Proceedings. First International Symposium on 3D Data Processing Visualization and Transmission. IEEE, 540-543.

Lisa Kausch, Anna Hilsmann, and Peter Eisert. 2017. Template-based 3D non-rigid shape estimation from monocular image sequences. In Proceedings of the conference on Vision, Modeling and Visualization. Eurographics Association, 37-44.

Hao Li, Bart Adams, Leonidas J Guibas, and Mark Pauly. 2009. Robust single-view geometry and motion reconstruction. In ACM Transactions on Graphics (TOG), Vol. 28. ACM, 175.

Hao Li, Robert W Sumner, and Mark Pauly. 2008. Global correspondence optimization for non-rigid registration of depth scans. In Computer graphics forum, Vol. 27. Wiley Online Library, 1421-1430.

Nadia Robertini, Dan Casas, Helge Rhodin, Hans-Peter Seidel, and Christian Theobalt. 2016. Model-based outdoor performance capture. In 2016 Fourth International Conference on 3D Vision (3DV). IEEE, 166-175.

Oliver Schreer, Ingo Feldmann, Sylvain Renault, Marcus Zepp, Peter Eisert, and Peter Kauff. 2019. Capture and 3D Video Processing of Volumetric Video. In Proc. IEEE International Conference on Image Processing (ICIP), Taipei, Taiwan.

Olga Sorkine and Marc Alexa. 2007. As-rigid-as-possible surface modeling. In Symposium on Geometry processing, Vol. 4. 109-116.

Olga Sorkine, Daniel Cohen-Or, Yaron Lipman, Marc Alexa, Christian Rössl, and H-P Seidel. 2004. Laplacian surface editing. In Proceedings of the 2004 Eurographics/ACM SIGGRAPH symposium on Geometry processing. ACM, 175-184.

Robert W Sumner, Johannes Schmid, and Mark Pauly. 2007. Embedded deformation for shape manipulation. In ACM Transactions on Graphics (TOG), Vol. 26. ACM, 80.

Gary K L Tam, Zhi-Quan Cheng, Yu-Kun Lai, Frank C Langbein, Yonghuai Liu, David Marshall, Ralph R Martin, Xian-Fang Sun, and Paul L Rosin. 2013. Registration of 3D point clouds and meshes: a survey from rigid to nonrigid. IEEE transactions on visualization and computer graphics 19, 7 (2013), 1199-1217.

Kiran Varanasi, Andrei Zaharescu, Edmond Boyer, and Radu Horaud. 2008. Temporal surface tracking using mesh evolution. In European conference on computer vision. Springer, 30-43.

Shih-En Wei, Jason Saragih, Tomas Simon, Adam W Harley, Stephen Lombardi, Michal Perdoch, Alexander Hypes, Dawei Wang, Hernan Badino, and Yaser Sheikh. 2019. VR facial animation via multiview image translation. ACM Transactions on Graphics (TOG) 38, 4 (2019), 67

Michael Zollhöfer, Matthias Nießner, Shahram Izadi, Christoph Rehmann, Christopher Zach, Matthew Fisher, Chenglei Wu, Andrew Fitzgibbon, Charles Loop, Christian Theobalt, and Others. 2014. Real-time non-rigid reconstruction using an RGB-D camera. ACM Transactions on Graphics (TOG) 33, 4 (2014), 156. 